\title{
BMJ Open Why do general practitioners prescribe antibiotics for upper respiratory tract infections to meet patient expectations: a mixed methods study
}

\author{
Stephanie Fletcher-Lartey, ${ }^{1}$ Melissa Yee, ${ }^{2}$ Christina Gaarslev, ${ }^{3}$ Rabia Khan ${ }^{4}$
}

To cite: Fletcher-Lartey S, Yee M, Gaarslev C, et al. Why do general practitioners prescribe antibiotics for upper respiratory tract infections to meet patient expectations: a mixed methods study. BMJ Open 2016;6:e12244

doi:10.1136/bmjopen-2016012244

- Prepublication history for this paper is available online. To view these files please visit the journal online (http://dx.doi.org/10.1136/ bmjopen-2016-012244).

Received 12 April 2016 Revised 8 July 2016 Accepted 9 August 2016

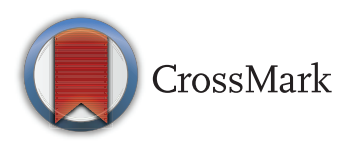

${ }^{1}$ Population Health, South Western Sydney Local Health District, Liverpool, New South Wales, Australia ${ }^{2}$ Evaluation, NPS

MedicineWise, Sydney, New South Wales, Australia

${ }^{3}$ Oslo kommune Helseetaten, Oslo, Norway

${ }^{4}$ Centre for Primary Health

Care and Equity, University of New South Wales, Sydney, New South Wales, Australia

Correspondence to

Rabia Khan;

rabia.khan@unsw.edu.au

\section{ABSTRACT}

Objectives: To describe the role patient expectations play in general practitioners (GPs) antibiotic prescribing for upper respiratory tract infections (URTI).

Methods: Concurrent explanatory mixed methods approach using a cross-sectional survey and semistructured interviews.

Settings: Primary care GPs in Australia. Participants: $584 \mathrm{GPs}$ (response rate of $23.6 \%$ ) completed the cross-sectional survey. 32 GPs were interviewed individually.

Outcome measure: Prescribing of antibiotics for URTI.

Results: More than half the GP respondents to the survey in Australia self-reported that they would prescribe antibiotics for an URTI to meet patient expectations. Our qualitative findings suggest that 'patient expectations' may be the main reason given for inappropriate prescribing, but it is an all-encompassing phrase that includes other reasons. These include limited time, poor doctor-patient communication and diagnostic uncertainty. We have identified three role archetypes to explain the behaviour of GPs in reference to antibiotic prescribing for URTIs. The main themes emerging from the qualitative component was that many GPs did not think that antibiotic prescribing in primary care was responsible for the development of antibiotic resistance nor that their individual prescribing would make any difference in light of other bigger issues like hospital prescribing or veterinary use. For them, there were negligible negative consequences from their inappropriate prescribing. Conclusions: There is a need to increase awareness of the scope and magnitude of antibiotic resistance and the role primary care prescribing plays, and of the contribution of individual prescribing decisions to the problem of antibiotic resistance.

\section{INTRODUCTION}

The misuse of antibiotics in primary care is a major contributor to antibiotic resistance. Upper respiratory tract infections (URTI) are
Strengths and limitations of this study

- The use of concurrent mixed methods provided a more comprehensive insight into the prescribing practices of antibiotics for an upper respiratory tract infection in primary care.

- There was a good representation of practice types, age, gender, location of general practitioners in the survey and the interviews. Younger general practitioners were underrepresented in the survey.

- It is difficult to ascertain whether general practitioners who did not participate in either the survey or the semistructured interview would have different prescribing patterns.

common presentations seen in general practice. URTI without complication (acute URTI or the 'common cold') is most often caused by a virus. Antibiotics have no efficacy in the treatment of viral infections, but are nevertheless often prescribed for their treatment. In Australia, the prescription rate for oral antibiotics most commonly used to treat acute URTI was 295 per 1000 people in 2013-2014. ${ }^{1}$ For the same period, the proportion of general practitioners (GPs) encounters for the management of acute URTI where systemic antibiotics were prescribed or supplied was $30.5 \%$. $^{1}$ There has been little change over the last 5 years in both statistics.

In Australia, NPS MedicineWise (NPS) develops health promotion interventions for health professionals and the community to influence the safe and judicious use of antibiotics. In 2012, a 5-year programme was implemented to encourage prudent use of antibiotics in the community. Mid-term evaluation of the programme revealed that, despite a high level of knowledge about antibiotic resistance, inappropriate antibiotic prescribing for URTI remains high in primary care. 
Patient/carer expectations for antibiotics have been identified by the health professionals who participated in the programme as the most significant barrier to antimicrobial stewardship (Fletcher-Lartey S, Yee M, Khan R. Participating in a clinical audit improves antibiotic stewardship but some challenges remain. Submitted for publication). Patient expectations or perceived expectations for antibiotics have been shown by others to influence the prescribing behaviour of doctors for respiratory conditions, more specifically for URTIs. ${ }^{2-5}$

Some patients merely expect some attention and a diagnosis from the physician. On the other hand, patients and carers expect a certain standard of care which comes with an expectation that the physician will provide medication such as antibiotics that will solve their problems. ${ }^{6}{ }^{7}$ Misconceptions regarding the indications for antibiotic use and lack of awareness of the benefits or harms associated with antibiotics have resulted in patients demand on physicians to provide them for respiratory tract infections. ${ }^{8}{ }^{9}$ It has been widely discussed that dissuading GPs from over prescribing is difficult. It has been said that GPs feel they have a duty of care to support their patients, because they feel that the patient expects them to 'do something'. ${ }^{9-11}$

Despite consistent and continued education and an associated high level of knowledge around antibiotic resistance, antibiotic prescribing rates for URTIs remain high in general practice. Further understanding of the underlying factors and circumstances that influence GP prescribing and the role of patient expectations is needed to inform the development of interventions to support antimicrobial stewardship and reduce inappropriate antibiotic prescribing.

The aim of this study was to explore the management of URTI and antibiotic prescribing in general practice in Australia. The purpose specifically was to understand and quantify the role of patients' expectations in respect to the prescribing of antibiotics for a URTI in order to inform the development of further educational interventions to reduce inappropriate prescribing of antibiotics.

\section{METHODS}

A concurrent explanatory mixed methods design was used as it was considered the most appropriate method to answer the research question. The quantitative phase of the study comprised a routine biannual survey to assess the extent to which patient expectations influence prescribing of antibiotics for URTI. The qualitative phase of the study comprised semistructured interviews to understand the influence of patient expectations on prescribing of antibiotics for URTI in general practice. The study was conducted in Australia and the fieldwork was conducted between May and August 2014.

\section{QUANTITATIVE}

A biannual survey of practicing GPs is conducted by NPS MedicineWise to evaluate its effectiveness in improving the quality use of medicines and medical tests in Australia. For the 2014 National GP Survey, a stratified random sample of anonymised 2500 GPs was drawn from a commercial medical publishing list. ${ }^{12}$ The sample was stratified by state and by geographical location to ensure sufficient representation of GPs who work in rural and remote areas. Sample size was equivalent to $10 \%$ of the total national GP workforce.

In May 2014, paper-based questionnaires were mailed to participants, together with details of alternative online completion. GPs in the sample with an email address listed were also emailed a link to the online questionnaire $(n=1000)$. GPs were requested to respond within 4 weeks, during which non-responding GPs were sent two reminders, at 2-week intervals. The entire questionnaire took about 15 min to complete; encompassing 11 question areas with a total of 30 questions. The survey instrument was pretested with five GPs unaffiliated with the research team and modified iteratively to improve clarity, face validity and content validity.

The outcome measure of interest for this study was the response to 'In your experience, how often do you prescribe antibiotics to meet patients' expectations when presenting with URTI?'. A Likert scale of five points ranging from never to very often was provided for responses. Descriptive statistics were used to summarise participants' data. The outcome measure was collapsed into a binary variable (sometimes, often and very often) and (never, rarely). Logistic regression analyses were used to investigate characteristics that could be associated with the likelihood of prescribing to meet expectations.

\section{QUALITATIVE}

\section{Recruitment and sample selection}

A qualitative approach involving semistructured interviews with GPs across Australia was employed. A purposive sample approach was used to select participants to include a diverse range of clinical practices.

In total, 30 GPs were considered to be sufficient to provide a variety in the sample with respect to geography, patient characteristics, age, sex and familiarity with NPS programmes and to achieve data saturation. The GPs were stratified by geographic location (urban/ rural) and practice socioeconomic profile (high/low).

The sample was selected from three cohorts. The first cohort was GPs who participated in a previous antibiotics educational programme and provided their contact details in an evaluation survey for the previous programme, indicating their willingness to participate in follow-up interviews. All 42 GPs who provided their details were contacted. From this group, 14 GPs participated.

The second cohort was GPs who participated in other NPS educational programmes but not in the antibiotic resistance programme in the last 5 years. A random sample of 150 was selected from this group. The third 
cohort was GPs registered with the commercial medical publishing house mail list. A random sample of 300 was selected from this group.

A stratified random sample of 172 GPs from the list of 450 GPs in the second and third cohort was initially contacted by mail, with a letter inviting them to participate in the study via telephone or face to face. Follow-up telephone calls were made 1 week after the letters were mailed, resulting in 35 GPs consenting to participate in the study. Eventually, three GPs were unable to commit to interviews during the study period, hence 32 interviews were completed (refer to figure 1).

\section{Data collection}

The semistructured interview guide was informed by a literature review and previous evaluation findings and was piloted with two GPs. There were three key areas and the questions explored the nature of the practice, management of URTI, factors influencing their prescribing and any influences of patient expectations on antibiotic prescribing. Our study sample for the qualitative component was $20 \mathrm{GPs}$ practicing in an urban practice (14 in high socioeconomic areas and 6 in low socioeconomic areas) and 12 GPs in a rural practice (4 in high socioeconomic areas and 8 in low socioeconomic areas). Telephone interviews were conducted with out of state, rural and remote participants, and five face to face interviews were conducted with GPs in Sydney metropolitan area. Informed consent was provided and interviews audio recorded with participants' consent. The interviews lasted on average, 20-30 $\mathrm{min}$ and were conducted by one author (SF-L) who is an experienced qualitative researcher with a background in conducting interviews and focus groups with health professionals.

\section{Data analysis}

Interviews were audiotaped, transcribed and analysed according to the methods of framework analysis. The research team agreed that the framework analysis approach was suitable for this study, as the interconnected stages of the systematic data analysis facilitated interpretation of participants' experiences and views from the development of descriptive to explanatory accounts; while presenting these views transparently. Framework analysis involves a systematic process of 'sifting, charting and sorting material according to key issues and themes' and allows for comparisons between and within cases, as well as sharing and discussion of data. Analysis involved familiarisation, development of a thematic framework, indexing, charting and interpretation. Interpretation involved thematic analysis, typologies and explanatory analysis. ${ }^{13} 14$

The interviews were coded by (SF-L) and reviewed by other team members (RK, MY) to allow for an assessment of coding validity. NVivo qualitative software was used to assist with coding and data management. Special attention was paid to any notable variations and divergences in perspectives between respondents from urban versus rural areas, participation in the educational programmes and from different socioeconomic practice settings.

\section{Cohort 1}

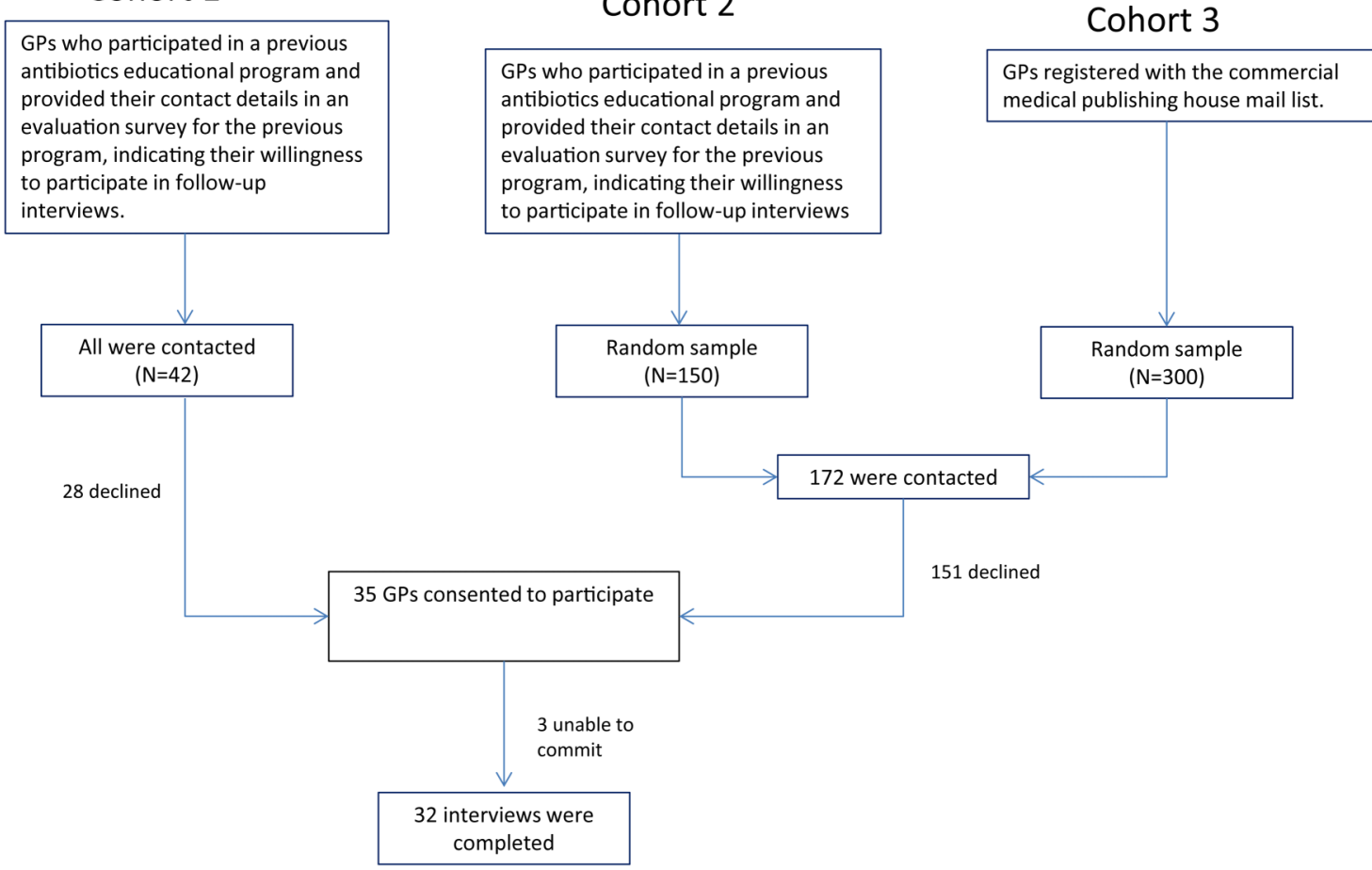

Figure 1 Qualitative study logistics. GP, general practitioner. 


\section{RESULTS}

\section{Quantitative}

The total response rate for the survey was $23.6 \%$. Of the total completed responses $(n=584), 82 \%$ were from the paper-based survey and $18 \%$ were from the online survey. The demographics of the respondents is presented in table 1 and is similar to the national workforce except that our survey was significantly underrepresented in those under 45 years as well as a significantly lower proportion of remote and very remote GPs.

A total of $56.5 \%$ GPs reported they would very often, often or sometimes prescribe antibiotics for URTI to meet patient expectations (table 2). The regression analysis assessed the influence of age, years worked as a GP, gender, location of practice and socioeconomic profile of practice population. Statistical analysis showed no significant predictors for prescribing.

\section{Qualitative}

Based on emerging themes, the concept of patient expectations and how these influence GP prescribing and the management of URTI in general practice were explored. We found there were no discernible differences in participants' experiences between the urban and rural settings, whether they participated in the educational programmes or if their practices were from varying socioeconomic settings.

\section{Patient expectations}

There was consensus among the participants that patients presenting with a URTI who visit GPs expected something from them, which could include a check-up, reassurance and guidance on symptomatic management, a medical certificate or antibiotics. These expectations have a subsequent impact on their prescribing practice.

Table 1 Demographics of survey respondents compared with the national GP workforce

\begin{tabular}{|c|c|c|}
\hline Characteristic & $\begin{array}{l}\text { Survey } \\
\text { respondents } \\
\text { N (\%) }\end{array}$ & $\begin{array}{l}\text { Australian GP } \\
\text { workforce } \\
\mathrm{N}(\%)^{29}\end{array}$ \\
\hline \multicolumn{3}{|l|}{ Gender } \\
\hline Male & $304(52.1 \%)$ & $18388(57 \%)$ \\
\hline Female & $272(47.2 \%)$ & $14013(43 \%)$ \\
\hline \multicolumn{3}{|c|}{ Remoteness area classification } \\
\hline Major city & $388(70.1 \%)$ & $21498(66 \%)$ \\
\hline Regional areas & $153(27.7 \%)$ & $9544(29 \%)$ \\
\hline $\begin{array}{l}\text { Remote and very } \\
\text { remote areas }\end{array}$ & $12(2.1 \%)$ & $1359(4 \%)$ \\
\hline \multicolumn{3}{|l|}{ Age group (years) } \\
\hline$<45$ & $138(24.3 \%)$ & $11,995(37 \%)$ \\
\hline $45-54$ & $173(30.4 \%)$ & $8515(26 \%)$ \\
\hline$>54$ & $258(45.4 \%)$ & $11891(37 \%)$ \\
\hline \multicolumn{3}{|c|}{ Years in practice (years) } \\
\hline $0-5$ & $67(11.5 \%)$ & NA \\
\hline $6-15$ & $131(22.4 \%)$ & NA \\
\hline$\geq 16$ & $368(63.1 \%)$ & NA \\
\hline
\end{tabular}

Table 2 Number of GPs who reported how often they prescribed antibiotics for URTI to meet patient expectations

\begin{tabular}{lr}
\hline $\begin{array}{l}\text { Response to the question 'In your } \\
\text { experience, how often do you prescribe } \\
\text { antibiotics to meet patients' expectations } \\
\text { when presenting with upper respiratory }\end{array}$ \\
$\begin{array}{lc}\text { Nract infection?' } & \text { Number of } \\
\text { GPs (\%) }\end{array}$ \\
\hline Very often & $8(1.5)$ \\
Often & $42(7.8$ \\
Sometimes & $255(47.2$ \\
Rarely & $215(39.7)$ \\
Never & $21(4)$ \\
\hline GP, general practitioner; URTI, upper respiratory tract infection.
\end{tabular}

While all patients are believed to be expecting something, only some patients are considered to be expecting antibiotics, and among these, a subset demands antibiotics. Participants estimated that, among patients with URTIs, $10-30 \%$ demanded antibiotics, and as a result they reported feeling pressured to prescribe antibiotics inappropriately. While the majority of participants shared this view, there were some who felt it was merely a perception of their colleagues.

The words 'expectation', 'demand' and 'pressure', were frequently used in reference to what patients desired from their GP visit. However, variations and divergences in the use of these terms were observed. It was deduced that the words were used in the following three ways:

1. Expectation indicating what a GP perceives the patient wants to happen or thinks will happen. Expectation was for 'something' which could be a prescription or something else like a medical certificate or reassurance, but the patient hasn't asked for antibiotics explicitly.

2. Expectation resulting in some patients demanding antibiotics resulting in GPs feeling pressured to prescribe. However, this pressure to prescribe can be real or perceived as a result of direct demands or as a result of the GP's perception. An example of the way in which the term 'expectation' was used in this regard is represented by the following statements.

'Well most of the time I will have to survive with my practice, so patient's expectation is a big thing for me. I cannot make them angry and drive them away, [laughing] I mean I will be out of my job next day, so I will have to follow the trend' (GP \#14).

3. Pressure and demand are sometimes used interchangeably to represent the direct or perceived action of patients in requesting antibiotics when they are not necessary.

The terms also implied that patients demand antibiotics, and GPs feel pressured to prescribe. Expectation was also used synonymously with demand and pressure in some cases. 


\section{Who expects antibiotics?}

High 'demand' patients

The participants reported that the majority of patients they see do not explicitly demand antibiotics. However, they did find it very difficult to convince the highdemand patients that they don't need antibiotics for URTIs. According to most GPs interviewed, only 10-30\% of patients fall in this category. For example:

Especially the ones that say, Doc... it's happened to me before, nothing new. I had pneumonia last year. I was in bad shape. I spent a few weeks in the hospital. This time I have the same symptoms as last year. I listen to them and say...It obviously shook you up a bit and you are very concerned about it. They will say, we are very concerned and we'd like antibiotics. Those patients, I call them demanding patients... (GP \#17)

Based on descriptions by the participants, it was suggested that 'high demand' for antibiotics patients were mainly parents with young children, recent migrants or people of culturally and linguistically diverse (CALD) backgrounds, which could include people with poor English skills and lower literacy levels.

Parents with young children were the group most frequently mentioned for placing demand for antibiotics on participants. It was indicated that parents were usually concerned about their child's suffering, worried about staying up all night with sick kids and concerned about going back to work, and hence expected antibiotics.

Recent migrants or people of CALD backgrounds were the second group most frequently identified as asking for antibiotics for a URTI. However, migrants were not identified as a homogenous group, as they could be either one of or a combination of persons with poor English skills, low educational or literacy levels and recently arrived migrants. Participants felt that some of these migrant groups were accustomed to obtaining antibiotics over the counter in their home countries.

\section{High 'need' patients}

Some patients were considered to be in need of antibiotics, and hence, this required prescribing of antibiotics. These patients include older patients with chronic conditions or history of pneumonia, chronic obstructive pulmonary disease (COPD) and diabetes; symptoms lasting longer than a week; persons going overseas requiring prophylaxis/or first aid medications; and most aboriginal patients. Appropriate prescribing was also described by several participants in rural areas, especially those based in the low socioeconomic settings that were faced with additional issues of availability and access to healthcare.

\section{Dealing with patients' expectations}

Consumer education (discussion/explanation) was the most common strategy reported by participants to manage patients' expectations and demands for antibiotics. Education was often given after expectations were identified and often in response to patients' demands. Common approaches included talking to the patient and providing the patient with information such as print-offs or handouts. Some participants used personalised written information and communicated with patients in their own language as strategies to deal with patients' expectations.

Some of the key educational messages delivered by participants include:

- 'URTI is likely to be a viral infection, and that antibiotics are only necessary for bacterial infections'.

- 'Antibiotics are not needed to treat URTIs, and they may not make you feel better any sooner'.

Several barriers to effective patient communication and education were identified by participants including limited time with patients to explain, limited educational resources that met the needs of specific patient groups and communication barriers (such as patients' poor English language skills, low literacy and cultural barriers to communication).

Delayed prescribing was reported to be widely used, but variations in practice suggests there is limited understanding of its effectiveness. The main reason cited by participants for issuing a delayed prescription, regardless of remoteness and socioeconomic setting, was to appease patients who demanded an antibiotic. For some, this was considered a compromise for patients who were considered to be very demanding and/or anxious. It was mentioned as a safety net for the patient in the event complications develop due to deterioration of their illness as well as a safeguard against any clinical malpractice and adverse outcomes should the patient develop any complications as a result of their illness.

\section{Prescribing patterns}

Most participants reported that they were more likely to prescribe antibiotics for URTIs, for one or more reasons. The persistent barriers reported about inappropriate antibiotic prescribing, other than patient expectations, included time pressure and diagnostic uncertainty. An issue reported from busier practices was insufficient time with each patient. Another concern expressed by participants was about medical liability in the event that antibiotics are not prescribed and there is a bad outcome.

I have to admit you would occasionally cave in because you don't want to make the situation worse. You don't want make their health worse but you don't want to make the doctor patient relationship worse either. (GP \#2)

... Some people I have to give antibiotics, they're very sick, their temperature's up. So there, I'm not waiting for academic research.. Infection can kill; an antibiotic has never killed anybody. So I choose to prescribe antibiotic ... (GP \#5) 
Usually when you are pressured in time and there are other things and you're running late, you're lagging behind ... sometimes you cannot help it. (GP \#22)

A very small minority of participants mentioned antibiotic resistance as a factor when prescribing for URTIs. When prompted, there was an element of antibiotic prescribing in primary care that was considered not responsible for development of antibiotic resistance and that prescribing at the individual level would not contribute either in light of other bigger issues like hospital prescribing or veterinary use.

Antibiotic resistance is not a problem when you look at community prescription patterns. For us GPs, its not a big issue. (GP \#14)

The empirical data collected in this study support a typology of three GP archetypes in regard to antibiotic prescribing for URTI: the 'compromising GP', the 'delaying GP' and the 'withholding GP'. Although the three role types are substantiated by the data, it is important to state that there is a continuum within each role, with 'grey areas' located at the boundaries of each role type.

\section{Antibiotics compromising}

This is the group of GPs who are more likely to prescribe antibiotics for an URTI for a whole range of reasons. These include limited consultation time, preserving GP-patient relationships, protecting business, inability to effectively negotiate or explain about antibiotics, empathy for patients and risk perception about the seriousness of the illness.

I have to admit you would occasionally cave in because you don't want to make the situation worse. You don't want make their health worse but you don't want to make the doctor patient relationship worse either. (GP \#1 City HSES)

If they don't understand that then unwittingly I would say, well I do not agree but since you say (so), I will give it to you. As a doctor I have to keep both the things in mind. I have to avoid antibiotics and look after the concerns of the patient. Infection can kill; an antibiotic has never killed anybody. So I choose to prescribe... (GP \#11)

\section{Antibiotics delaying}

This group of GPs reported that they mainly explain to patients that they do not need antibiotics, but will provide the patients with a prescription to be used at a later date, in the event that their condition deteriorates. This is reportedly mainly for demanding patients as a means to appease patients. Additionally, delayed prescriptions are given to those patients whom GPs may be concerned that their symptoms may deteriorate over time.
... also because sometimes patients will say; "please I want antibiotics". I really have to get rid of this and then you actually (tell them), well you can't get rid of this because it is a virus. You have no choice; you just have to get better...So I tend to come to a compromise if they are really, really.., if I can't talk them out of it. I'll just (say) okay why not, I'll give you a script anyway. (GP \#8)

I also tend to use delayed prescriptions for antibiotics quite a lot. If a patient seems very keen on the idea of antibiotics and it is hard to dissuade away from them I would often send them with a prescription but with some quite specific advice about when to start the antibiotics. (GP \#23)

\section{Antibiotics withholding}

This group of GPs often indicated that they mainly explain to patients that they do not need antibiotics, and will not provide a script to patients, not even delayed prescribing. These GPs indicated that they would encourage symptomatic management, advise patients to return to the clinic should their condition deteriorate.

I just say this is what you need to do. Nothing else will work, like cough mixtures and stuff. Antibiotics aren't indicated and here, do this. Maybe I'm too directive. (GP \#3)

Yeah, we discuss their treatment options. If I think it's viral I'll explain to them why...If they don't like it they'll just go to another GP and get antibiotics so [laughs]. I have no idea who does it because they won't tell me, but I'm sure a lot of people do that. A lot of people go to several doctors to try and get antibiotics. They just want a prescription. There's a lot of pressure on GPs, I know that. I'm stubborn [laughs]. I educate them. (GP \#30)

\section{DISCUSSION}

Our study shows that more than half the GP respondents to the survey in Australia self-reported that they would prescribe antibiotics for an URTI to meet patient expectations. However, the interviews indicated that patient expectations is just one reason, and it actually reflects a more complex situation and a multitude of reasons which reflect the social circumstances under which they themselves work and underlying patient-related socioeconomic, situational and cultural factors. However, there might be an indication that most GPs are not cognisant of the role primary care prescribing plays in regard to antibiotic resistance. We have identified three role archetypes to explain the behaviour of GPs in relation to prescribing antibiotics for URTI. These role archetypes can be used to develop educational resources that cater for different preferences for the three groups. We also found, from a GP perspective, that a small group of patients explicitly ask them to prescribe antibiotics, but they are a heterogeneous subset of the population, influenced by sociocultural factors and personal needs. 


\section{Comparison with other studies}

Our findings are similar to other studies that suggest most GPs acknowledge that patient pressure or perceived pressure influences their decision to prescribe antibiotics. A recent survey of 1000 GPs in the UK, found that $55 \%$ felt under pressure, mainly from patients, to prescribe antibiotics, even if they were not sure that they were necessary, and $44 \%$ admitted that they had prescribed antibiotics to get a patient to leave the surgery. A similar proportion (45\%) had prescribed antibiotics for a viral infection, knowing that they would not be effective. ${ }^{15}$

Studies in North America and Europe have also reported that GPs are generally aware of, and concerned about, the broad issue of antimicrobial resistance. However, they frequently do not perceive it as a problem within their everyday practice or one which is linked to their prescribing practices. ${ }^{16-20}$ Antimicrobial resistance was considered more of a community public health issue whereas the GPs priority was the health of the individual patient. For clinicians, whose primary concern is the unwell individual, the impact of antimicrobial use on the prevalence of community-based resistance may not be considered of great importance. Several studies have indicated that other issues like previous experience, uncertainty about diagnosis, ease of follow-up and fear of consequences of non-prescribing, as well as perceptions of potential conflict with patients and perceived pressure to prescribe, and consequences for the future doctor-patient relationship are more of a concern for GPs prescribing antibiotics than antibiotic resistance ${ }^{21-25}$ Similar views regarding concerns about the consequences of not prescribing antibiotics and resistance being primarily a societal issue have been reported by GPs in other qualitative studies. ${ }^{18} 1926$

\section{Strengths and limitations of study}

Our main strength lies in the mixed methods approach we had chosen, to understand how and why patient expectations manifest in the prescribing of antibiotics for an URTI.

One of our limitations for the survey was that it is an administrative organisational survey, so only one question was asked about antibiotic prescribing. The survey covered a range of topics pertinent to primary care practice. Our response rate of $23.6 \%$ is reasonable for a health professional survey. In Australia, the response rates to surveys with medical practitioners are often below $30 \% .{ }^{27}$ Overall, our survey managed to closely match GP demographics although there was an under representation of younger GPs. There is some evidence to suggest that younger GPs are less likely to prescribe antibiotics for a URTI. ${ }^{28}$

The GPs spoke frankly about URTI consultations and often pointed out that their practice at times ran counter to research evidence. Such revelations of why GPs practise the way they do could not have been discerned from a survey or from directly observing medical encounters. We may have missed important data that were obtainable from those who did not participate. Our recruitment process ensured we obtained views from GPs practising in a range of practices. It is not clear whether our findings would generalise to clinicians in other countries where the medical culture and the approach to direct elicitation of expectations may differ; however, our aim was to identify important themes from the GP interviews rather than generating statistically representative data.

\section{Conclusions and policy implications}

Our findings suggest that antibiotic resistance does not form part of the GP's decision to prescribe antibiotics for URTI and other reasons such as patient expectations are more pertinent. These reasons are mostly centred on the complexity of the doctor-patient relationship. Single, simple solutions are therefore unlikely to change prescribing habits. The problem is a cultural one and goes beyond doctors simply not knowing of the evidence from clinical trials or guidelines. Different strategies are also needed in terms of GPs dealing with the 'high demand' patients as well as developing interventions targeting the needs of the high-demand patient group.

Twitter Follow Stephanie Fletcher-Lartey at @stephjewels and Rabia Khan at @rabiaikhan

Contributors SF-L designed the qualitative component of the study, collected and analysed the data, and drafted the manuscript. MY codesigned the study, analysed the quantitative component of the study and critically revised the manuscript. CG provided substantial contributions to the data acquisition and analysis of the quantitative component of the study, interpretation of the data and drafting of the manuscript. RK was a principal investigator on the study and contributed to the design of the study and analysis of the findings as well as to the writing of the manuscript.

Funding This research was funded by the Australian Department of Health through NPS MedicineWise. We also acknowledge the contribution of Vanessa Simpson and Margie Campbell for their advice during project development, and Suzanne Blogg and Lynn Weekes for their thoughtful contribution to editing this article.

Competing interests None declared.

Ethics approval Royal Australasian College of General Practice National Research and Evaluation Ethics Committee.

Provenance and peer review Not commissioned; externally peer reviewed.

Data sharing statement Extra data can be accessed via the Dryad data repository at http://datadryad.org/ with the doi:10.5061/dryad.gt76v.

Open Access This is an Open Access article distributed in accordance with the Creative Commons Attribution Non Commercial (CC BY-NC 4.0) license, which permits others to distribute, remix, adapt, build upon this work noncommercially, and license their derivative works on different terms, provided the original work is properly cited and the use is non-commercial. See: http:// creativecommons.org/licenses/by-nc/4.0/

\section{REFERENCES}

1. SCRGSP (Steering Committee for the Review of Government Service Provision). Report on Government Services 2015. Canberra: Health, Productivity Commission, 2015.

2. Sung L, Arroll J, Arroll B, et al. Antibiotic use for upper respiratory tract infections before and after a education campaign as reported by general practitioners in New Zealand. $N Z$ Med $J$ 2006;119:U1956. 
3. Mangione-Smith R, Elliott MN, Stivers T, et al. Ruling out the need for antibiotics: are we sending the right message? Arch Pediatr Adolesc Med 2006;160:945-52.

4. Bauchner H, Pelton SI, Klein JO. Parents, physicians, and antibiotic use. Pediatrics 1999;103:395-401.

5. Little P, Williamson I, Warner G, et al. Open randomised trial of prescribing strategies in managing sore throat. $B M J$ 1997;314:722-7.

6. Lewis PJ, Tully MP. The discomfort caused by patient pressure on the prescribing decisions of hospital prescribers. Res Social Adm Pharm 2011;7:4-15.

7. Little $\mathrm{P}$, Dorward M, Warner G, et al. Importance of patient pressure and perceived pressure and perceived medical need for investigations, referral, and prescribing in primary care: nested observational study. BMJ 2004;328:444.

8. Belongia EA, Naimi TS, Gale CM, et al. Antibiotic use and upper respiratory infections: a survey of knowledge, attitudes, and experience in Wisconsin and Minnesota. Prev Med 2002;34:346-52.

9. Del Mar C, Glasziou P, Lowe JB, et al. Addressing antibiotic resistance: focusing on acute respiratory infections in primary care. Aust Fam Physician 2012;41:839.

10. Doust J, Del Mar C. Why do doctors use treatments that do not work? BMJ 2004;328:474-5.

11. White C. If it doesn't work, stop it: summary of rapid responses. BMJ 2004;328:1016.

12. Australasian Medical Publishing Company Direct. Doctor and healthcare lists. http://www.ampcodirect.com.au

13. Smith J, Firth J. Qualitative data analysis: the framework approach. Nurse Res 2011;18:52-62.

14. Pope C, Ziebland S, Mays N. Qualitative research in health care: analysing qualitative data. BMJ 2000;320:114-16.

15. Cole A. GPs feel pressurised to prescribe unnecessary antibiotics, survey finds. BMJ 2014;349:g5238.

16. Simpson SA, Wood F, Butler CC. General practitioners' perceptions of antimicrobial resistance: a qualitative study. J Antimicrob Chemother 2007;59:292-6.

17. Kumar S, Little P, Britten N. Why do general practitioners prescribe antibiotics for sore throat? Grounded theory interview study. BMJ 2003;326:138.
18. Butler CC, Rollnick S, Pill R, et al. Understanding the culture of prescribing: qualitative study of general practitioners' and patients' perceptions of antibiotics for sore throats. BMJ 1998;317:637-42.

19. Wood F, Simpson S, Butler CC. Socially responsible antibiotic choices in primary care: a qualitative study of GPs' decisions to prescribe broad-spectrum and fluroquinolone antibiotics. Fam Pract 2007;24:427-34.

20. Paluck E, Katzenstein D, Frankish CJ, et al. Prescribing practices and attitudes toward giving children antibiotics. Can Fam Physician 2001;47:521-7.

21. Tonkin-Crine S, Yardley L, Little P. Antibiotic prescribing for acute respiratory tract infections in primary care: a systematic review and meta-ethnography. J Antimicrob Chemother 2011;66:2215-23.

22. Lucas PJ, Cabral C, Hay AD, et al. A systematic review of parent and clinician views and perceptions that influence prescribing decisions in relation to acute childhood infections in primary care. Scand J Prim Health Care 2015;33:11-20.

23. Cockburn J, Pit S. Prescribing behaviour in clinical practice: patients expectations and doctors' perceptions of patients' expectations--a questionnaire study. BMJ 1997;315:520-3.

24. Stivers T, Manione-Smith R, Elliott MN, et al. Why do physicians think parents expect antibiotics? What parents report vs what physicians believe. J Fam Pract 2003;52:140-8.

25. Ashdown HF, Räisänen U, Wang K, et al. Prescribing antibiotics to 'at-risk' children with influenza-like illness in primary care: qualitative study. BMJ Open 2016;6:e011497.

26. Bjorkman I, Erntell M, Roing M, et al. Infectious disease management in primary care: perceptions of GPs. BMC Fam Pract 2011:12:1.

27. Britt $\mathrm{H}$, Miller GC, Charles J, et al. General practice activity in Australia 2008-09. BEACH: Bettering the Evaluation and Care of Health. General Practice Series No.25. Cat No. GEP 25. Canberra: AlHW, 2009.

28. Cadieux G, Tamblyn R, Dauphinee D, et al. Predictors of inappropriate antibiotic prescribing among primary care physicians CMAJ 2007;177:877-83.

29. Australian Government Department of Health and Aging. General Practice Workforce Statistics 1984-85 to 2013-14. Cnberra: Doha, 2014. 